\section{FACTEURS DE RISQUE D'UNE SÉROLOGIE TOXOPLASMIQUE POSITIVE CHEZ LA FEMME ENCEINTE AU MAROC}

LABOUDI M.*, EL MANSOURI B.*, SEBTI F.*, AMARIR F.*, COPPIETERS Y.** \& RHAJAOUI M.*

Monsieur,

Au Maroc, bien que très peu d'études aient été réalisées sur la séroprévalence de la toxoplasmose, aucune évaluation des facteurs de risque n'a été publiée à ce jour. Mekouar, en 1972, avait estimé la séroprévalence de la toxoplasmose à $51,5 \%$. Une prévalence similaire de 50,6\% a été rapportée par El Mansouri et al. (2007) dans la région de Rabat. Ce taux est élevé par comparaison aux $32 \%$ rapportés par Chouchane et al. dans leur étude préliminaire réalisée dans la région de Sétif (Algérie).

Plusieurs facteurs interviennent dans la contamination toxoplasmique et leur maitrise contribue à prévenir la maladie. L'objectif de notre étude a donc été de déterminer les facteurs de risque impliqués dans l'acquisition de cette infection. Cette étude transversale à visée analytique a porté sur 1020 femmes enceintes, adressées par leurs médecins généralistes et gynécologues à l'Institut National d'Hygiène de Rabat, dans le cadre d'un suivi sérologique pendant la grossesse. L'échantillonnage a été effectué d'une façon systématique et sa taille a été calculée en fonction d'une prévalence théorique, avec un intervalle de confiance de $95 \%$ et un niveau de risque d'erreur de $5 \%$. Durant la période d'étude, chaque femme a répondu volontairement au questionnaire portant sur l'âge, la consommation de viande mal cuite, le niveau d'étude, les connaissances sur la toxoplasmose, le contact avec la terre (jardinage, activités agricoles) et les chats. La recherche des anticorps anti Toxoplasma a été réalisée chez toutes ces femmes par la technique ELISA (Biorad, France) et les titres en IgG ont été exprimés en unités internationales (UI/ml).

L'analyse statistique des résultats a été faite à l'aide du logiciel Epiinfo 2000. Après ajustement des rapports, une analyse bivariée a servi à comparer les variables

\footnotetext{
* Département de parasitologie, Institut national d'hygiène, Rabat, Maroc.

** Département d'épidémiologie et de promotion de la santé, École de Santé Publique, ULB, Bruxelles, Belgique.

Correspondance : Majda Laboudi, Département de parasitologie, Institut National d'Hygiène, BP 769, avenue Ibn Batouta, Rabat - Agdal, Rabat, Maroc.

Tél.: + 21214635765 - Fax : + 21237772067.

E-mail : lamajda@yahoo.fr
}

entre elle. L'association entre le statut sérologique toxoplasmique et les facteurs de risques déterminant cette infection a été analysée par l'utilisation du $\mathrm{Chi}^{2}$ de Pearson et des odds ratio (OR) avec un intervalle de confiance (IC) de $95 \%$.

Sur les 1020 femmes enceintes ayant participé à cette étude, 50,6\% présentaient une sérologie de toxoplasmose positive (El Mansouri et al., 2007). La séroprévalence augmente linéairement avec l'âge : de 32,4\% chez les femmes de moins de 20 ans jusqu'à 63,8 \% chez les femmes de plus de 40 ans (figure 1). La fréquence d'une sérologie toxoplasmose positive est significativement plus élevée chez les femmes de plus de 40 ans que chez celles de moins de 20 ans ( $\mathrm{p}<0,05)$.

L'analyse bivariée des facteurs de risque a mis en évidence une association statistiquement significative entre le contact avec le sol, le bas niveau scolaire, l'absence de connaissance sur la toxoplasmose et une sérologie positive $(p<0,05)$. Aucune différence statistique n'a été trouvée entre la consommation de viande crue, la possession d'un chat et l'infection toxoplasmique (tableau I). Par ailleurs, le niveau de connaissance du mode de transmission est meilleur chez les femmes séropositives $(66,7 \%)$ que chez les femmes non immunisées. Cette association est statistiquement significative. Des résultats similaires ont été observés dans une étude réalisée par Jumaian en 2005 en Jordanie. En plus, ce facteur ne constitue ni un facteur de confusion ni un effet modificateur quand il est associé avec un faible niveau d'étude qui constitue un risque de contracter la maladie $(\mathrm{OR}=0,59 ; \mathrm{IC}=1,25-2,26 ; \mathrm{p}<0,05)$. De même, le contact avec la terre (jardinage et activités agricoles) pourrait aussi contribuer à l'acquisition du parasite. Birgisdottir et al. (2006) ont constaté également que le contact avec la terre constituait le facteur principal de risque d'infection par T. gondii.

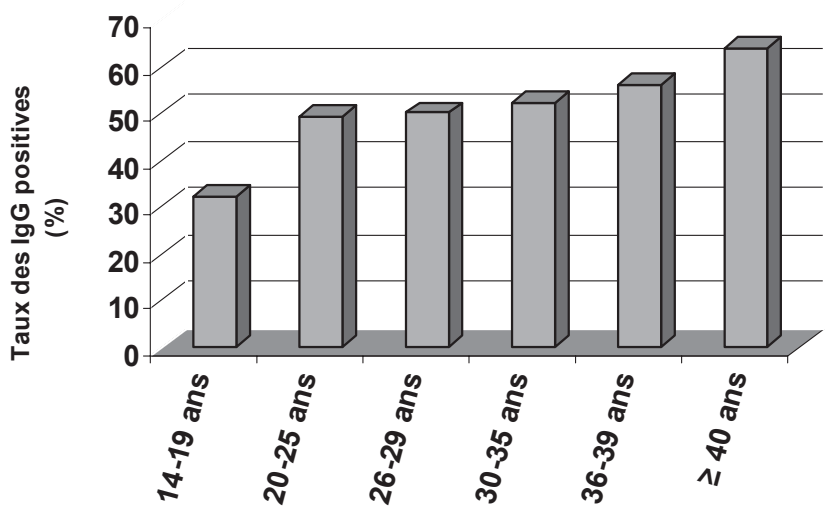

Fig. 1. - Séroprévalence de la toxoplasmose par tranches d'âge. 


\begin{tabular}{|c|c|c|c|c|c|c|}
\hline Variables & & $\begin{array}{c}\text { Séropositives } \\
\%(\mathrm{~N})\end{array}$ & $\begin{array}{c}\text { Séronégatives } \\
\%(N)\end{array}$ & $\mathbf{p}$ & OR & IC $95 \%$ \\
\hline Contact avec le sol & $\begin{array}{l}\text { Oui } \\
\text { Non }\end{array}$ & $\begin{array}{l}54,05(200) \\
44,61(290)\end{array}$ & $\begin{array}{l}45,95(170) \\
55,39(360)\end{array}$ & 0,003 & 1,46 & $1.12-1.90$ \\
\hline Niveau d'éducation & $\begin{array}{l}\text { Analphabètes } \\
\text { Primaire }\end{array}$ & $\begin{array}{l}57,69(150) \\
44,73(340)\end{array}$ & $\begin{array}{l}42,31(110) \\
55,27(420)\end{array}$ & 0,0003 & 1,68 & $1,25-2,26$ \\
\hline Connaissance sur la maladie & $\begin{array}{l}\text { Oui } \\
\text { Non }\end{array}$ & $\begin{array}{l}66,66(40) \\
46,87(450)\end{array}$ & $\begin{array}{c}33,34(20) \\
53,13(510)\end{array}$ & 0,003 & 0,44 & $0,24-0,79$ \\
\hline Consommation de viande mal cuite & $\begin{array}{l}\text { Oui } \\
\text { Non }\end{array}$ & $\begin{array}{l}50 \quad(160) \\
47,14(330)\end{array}$ & $\begin{array}{ll}50 & (160) \\
52,86 & (370)\end{array}$ & NS & 1,12 & $0.85-1.47$ \\
\hline Contact avec le chat & $\begin{array}{l}\text { Oui } \\
\text { Non }\end{array}$ & $\begin{array}{l}51,42(180) \\
46,26(310)\end{array}$ & $\begin{array}{l}48,58(170) \\
53,74(360)\end{array}$ & NS & 1,23 & $0.94-1.61$ \\
\hline Niveau d'hygiène & $\begin{array}{l}\text { Bas } \\
\text { Moyen }\end{array}$ & $\begin{array}{r}2,02(10) \\
47,52(480)\end{array}$ & $\begin{array}{cl}0 & (0) \\
52,48 & (530)\end{array}$ & & & \\
\hline
\end{tabular}

NS : non significatif.

Tableau I. - Analyse bivariée des facteurs influençant la séropositivité de la toxoplasmose chez les femmes.

La consommation de viande mal cuite (surtout de viande hachée et assaisonnée) n'apparaît pas dans notre étude comme un risque potentiel d'acquisition des anticorps toxoplasmiques. Un résultat identique a été rapporté dans une étude turque (Ertug et al., 2005). En revanche, en Algérie, Chouchane et al. (2008) ont montré une association significative entre la consommation de viande mal cuite et l'acquisition des anticorps toxoplasmiques. Enfin, dans notre étude, le contact direct avec un chat sans propriétaire n'est pas considéré comme un facteur important de risque. Dans d'autres études épidémiologiques, l'association entre le chat et la maladie reste difficile à évaluer, car c'est le sol et non pas le chat qui est directement impliqué dans la transmission de la toxoplasmose. Les oocystes ne se trouvent pas sur le pelage des chats, mais ils sont enfouis dans le sol avec leurs fèces. Un contact direct avec le sol a été trouvé associé avec la séropositivité de la toxoplasmose dans notre étude.

Cette étude permet de conclure, que le contact avec le sol et l'analphabétisme paraissent être les principaux facteurs de risque de contracter une toxoplasmose. Par ailleurs, les informations recueillies peuvent être utilisées pour la mise en place d'un dispositif obligatoire de dépistage et de surveillance sérologique des femmes enceintes séronégatives, dans un but préventif.

\section{RÉFÉRENCES}

Birgisdottir A., Asbjornsdottir H., CoOK E., Gislason D., JanssOn C., Olafsson I. et al. Seroprevalence of Toxoplasma gondii in Sweden Estonia and Iceland. Scandinavian Journal of Infectious Diseases, 2006, 38, 625-631.

Chouchane M., Baki C.A, TouabTi A. \& Laouami S. La toxoplasmose chez la femme enceinte à Sétif, étude préliminaire. Communication orale, $1^{\text {ères }}$ rencontres scientifiques Rennes-Sétif, Faculté de médecine, Université de Rennes, 7-11 novembre 2007 (http://1rsrs.univ-rennes1.fr/Communications/Medecine/Chouchane.pdf)

El Mansouri B., Rhajaoui M., Sebti F., Amarir F., Laboudi M., Bchitou R., Hamad M. \& Lyagoubi M. Séroprévalence de la toxoplasmose dans la ville de Rabat au Maroc. Bulletin de la Société de Pathologie Exotique, 2007, 100 (2), 113116.

JUMAIAN N.F. Seroprevalence and risk factors for Toxoplasma infection in pregnant women in Jordan. Eastern Mediterranean Health Journal, 2005, 11, 45-51.

Mekouar A. Contribution de l'épidémiologie de la toxoplasmose, sérologie de la toxoplasmose au Maroc, Thèse de médecine, Bordeaux, 1972.

Ertug S., Okyay P., Turkmen M. \& Yuksel H. Seroprevalence and risk factors for toxoplasma infection among pregnant women in Aydin province, Turkey. BMC Public Health, 2005, 5, 66.

Reçu le 11 octobre 2008 Accepté le 10 décembre 2008 